\title{
Cannabis d'hier et cannabis d'aujourd'hui. Augmentation des teneurs en THC de 1993 à 2004 en France
}

\section{Cannabis of the past and cannabis todays. Increase of THC contents from 1993 to 2004 in France}

Patrick MURA $^{(1) *}$, Bertrand BRUNET ${ }^{(1)}$, Laurence DUJOURDY ${ }^{(2)}$, Carole PAETZOLD $^{(2)}$, Geneviève BERTRAND ${ }^{(2)}$, Bruno SERA ${ }^{(3)}$, Benoît SACLIER ${ }^{(2)}$, Marc DEVEAUX ${ }^{(3)}$, Gilbert PÉPIN ${ }^{(3)}$, Martine PERRIN ${ }^{(4)}$, Yannick LECOMPTE ${ }^{(4)}$, Véronique DUMESTRE-TOULET ${ }^{(5)}$, Vincent CIRIMELE ${ }^{(6)}$, Pascal KINTZ ${ }^{(6)}$

(1) Laboratoire de Toxicologie et Pharmacocinétique, CHU, Poitiers

(2) Laboratoires de l'Institut National de Police Scientifique

(3) Laboratoire Toxlab, Paris

(4) Département Toxicologie, IRCGN, Rosny sous Bois

(5) Laboratoire Toxgen, Bordeaux

(6) Laboratoire Chem Tox, Illkirch

*Auteur à qui adresser la correspondance : Patrick MURA, Laboratoire de Toxicologie et Pharmacocinétique, Centre Hospitalier Universitaire, BP 577 - 86021 POITIERS - FRANCE Tél : +33549443923-Fax : +33549443834

(Reçu le 20 janvier 2006 ; accepté le 14 février 2006)

\section{RÉSUMÉ}

Une étude précédente, réalisée sur des échantillons de saisie en France, nous avait permis de montrer que la teneur en delta-9 tétrahydrocannabinol (THC) dans les échantillons de « résine » et « d'herbe » avait augmenté durant la période 1993-2000 et tout particulièrement depuis 1996.

L'objet de la présente étude a été de déterminer l'évolution de ces teneurs en THC pendant la période 2001-2004 et de comparer cette évolution avec celle de la période précéden-

\section{SUMMARY}

A previous study, performed on seized cannabis products from France, had allowed us to indicate that there has been an increase in the delta-9 tetrahydrocannabinol (THC) content from herbal products and resin samples during the period 1993-2000 and overall from 1996.

The purpose of the present study was to observe the pattern of the THC content of cannabis which was available in France between 2001 and 2004 and to compare these results 
te. Nous avons regroupé tous les résultats obtenus pendant cette période par les laboratoires de la Gendarmerie Nationale, de l'Institut National de Police Scientifique et de quatre laboratoires d'expertise en France, représentant 2613 échantillons de "résine » et 709 échantillons "d'herbe 》. Les analyses étaient réalisées par chromatographie gazeuse couplée à la spectrométrie de masse.

En ce qui conceme "l'herbe ", la très grande majorité des échantillons (71\%) contiennent moins de $5 \%$ de principe actif, ce qui peut être expliqué par des récoltes fréquemment effectuées avant maturité des plants. En revanche, près de l'échantillon sur 5 contient plus de $10 \%$ de THC.

Les résultats concenant la « résine » indiquent que, depuis 10 ans, le nombre d'échantillons à faible teneur en THC (inférieurs à $5 \%$ de principe actif) a diminué de manière très significative (passant de 48\% des échantillons en 1993 à $15 \%$ en 2004). Les échantillons contenant plus de $20 \%$ de THC restent très rarement rencontrés, représentant seulement $2 \%$ des saisies. L'évolution la plus caractéristique a concerné les échantillons de résine contenant entre 10 et $15 \%$ de THC. Cette catégorie n'a cessé d'augmenter depuis 1993, passant de 1\% des échantillons à 32\% en 2004.

Cette étude confirme que les teneurs en THC dans les échantillons de "résine " disponibles sur le marché clandestin français ont régulièrement augmenté depuis une dizaine d'années, ayant été en moyenne multipliées par 2 entre 1993 et 2004. Une telle observation n'est pas sans conséquence en terme de santé publique car l'utilisation de cannabis « haut dosage " peut être responsable d'hallucinations, d'attaques de panique ou autres états psychotiques aigus.

\section{MOTS-CLÉS}

Cannabis, teneurs, $T H C$, résine, herbe, produit de saisie.

\section{Introduction}

Parmi la soixantaine de cannabinoïdes présents dans Cannabis sativa var: indica, le $\Delta^{9}$-transtétrahydrocannabinol (THC) constitue le principal produit psychoactif chez l'homme.

Barnett et coll (1) ont étudié sur simulateurs de conduite les erreurs de conduite induites par une consommation de « joints » dosés à 100,200 ou $250 \mu \mathrm{g} / \mathrm{kg}$ de poids du sujet. Ils ont indiqué l'existence d'une corrélation linéaire significative pendant une durée de 2 à $7 \mathrm{~h}$ (selon les effets, le plus persistant étant le suivi de trajectoire). Harder et coll (2) ont comparé l'évolution dans le temps des concentrations sanguines de THC et les effets psychiques ressentis par les sujets, après consommation de « joints » contenant 1,3 ou $9 \mathrm{mg}$ de THC. Ils ont montré que l'amplitude des effets était dépendante de la dose. Plus récemment, Lane et coll (3) ont indiqué que les effets sur le rythme cardiaque et sur la mémoire de travail étaient d'autant plus significatifs que les doses utilisées étaient élevées.

De tous ces travaux, il résulte donc que les effets pharmacologiques du THC sur les fonctions cognitives et motrices chez un sujet donné seront d'autant plus to those of the previous study. We have collected all the results obtained from 2001 to 2004 on seized cannabis products (2613 resin samples and 709 herbal products) by the laboratories of national police force and from four forensic toxicology independent laboratories. The THC content was determined by gas chromatography - mass spectrometry. Most of herb samples. $(71 \%)$ contained less than $5 \%$ of THC. This result can be explained by the fact that harvestings are often made before ripeness. In tum, about one sample out of five contained more than $10 \%$ of THC.

About resin, the reached results indicated that during the last 10 years the number of low THC content samples (less than $5 \%$ of $\mathrm{THC}$ ) had decreased very significantly (48\% of samples in 1993 versus 15\% in 2004). Samples with more than $20 \%$ of THC were seldom found (only $2 \%$ of seizures). The most significant evolution concened samples containing between 10 and $15 \%$ of THC. This level has constantly increased since 1993 (around 1\% of samples analysed to $32 \%$ in 2004).

This study confirms that THC content of cannabis available in France has regularly increased during the past ten years (enhanced by a factor 2 between 1993 and 2004). Such findings may be consistent for public health because the use of such "high dosage" cannabis may be involved in the occurrence of hallucinations, panic attacks or other acute psychotic states.

\section{KEY-WORDS}

Cannabis, dosage, THC, cannabis resin, hashish, marijuana.

importants que le produit utilisế contient un pourcentage élevé en THC.

Des effets délétères très prononcés (états délirants aigus, hallucinations, crises de panique) sont de plus en plus souvent rapportés dans la littérature (4) et de nombreux auteurs $(5-7)$ expliquent ces observations par une augmentation très importante au cours de la dernière décennie des teneurs en THC dans les produits à base de cannabis disponibles en France. Une étude précédente avait été réalisée sur 5152 échantillons d'herbe et de résine de cannabis saisis en France de 1993 à 2000 (8). Les résultats avaient montré une augmentation des teneurs en THC depuis 1996 avec l'apparition de nouveaux produits très fortement titrés, mais que ces échantillons très concentrés ne correspondaient qu'à une minorité des produits disponibles sur le marché clandestin français. Une étude australienne, publiée en 2000, avait fait le même constat (9).

L'objectif de ce travail a été d'étudier l'évolution des teneurs en THC dans les produits à base de cannabis ayant été saisis en France pendant la période 20012004 et de comparer cette évolution à celle observée de 1993 à 2000. 


\section{Méthodes}

Nous avons rassemblé 3322 résultats d'analyses obtenus depuis 2001 sur des produits de saisies effectuées en France par les 5 laboratoires de l'Institut National de Police Scientifique (Lyon, Marseille, Lille, Toulouse, Paris), le laboratoire de I'Institut de Recherches Criminelles de la Gendarmerie Nationale (Rosny Sous Bois), le laboratoire Toxlab (Paris), le laboratoire Toxgen (Bordeaux), le laboratoire ChemTox (Illkirch) et le laboratoire de Toxicologie et Pharmacocinétique du Centre Hospitalier Universitaire de Poitiers. Les données transmises par les laboratoires de l'Institut National de Police Scientifique étaient extraites de la base S.T.U.P.S. (Système de Traitement Uniformisé des Produits Stupéfiants). Les renseignements fournis par tous les laboratoires indiquaient l'année de la saisie, le type de produit (herbe, résine, etc.) et la teneur observée en THC. Les analyses ont été réalisées par chromatographie en phase gazeuse couplée à la spectrométrie de masse et les résultats étaient exprimés en pourcentages par rapport au poids de matière sèche.

\section{Résultats}

Parmi les 3322 échantillons, 2613 concernaient de la « résine » et 709 étaient des échantillons « d'herbe » (principalement sommités florales, feuilles et «pollens »).

Les résultats concernant l'herbe sont indiqués dans la Figure 1. La très grande majorité des échantillons ( $71 \%$ ) contenaient moins de $5 \%$ de principe actif. Cette observation est cependant à considérer avec la plus grande réserve. En effet, les échantillons confiés aux experts pour analyse correspondent soit à des produits « finis », prêts à la consommation ou à la vente, soit à des saisies de récoltes « sur pied ». Dans ce dernier cas, les échantillons sont le plus souvent saisis par les forces de l'ordre avant que les plants ne soient à maturité, ce qui se traduit par des teneurs en THC pouvant être très faibles. Par ailleurs, pour une même saisie, les résultats peuvent être très variables selon que l'échantillon analysé correspond à un plant femelle ou mâle et selon la partie de la plante (fleurs ou inflorescences) (10). En revanche, près de $10 \%$ des échantillons d'herbe contenaient plus de $15 \%$ de principe actif dont $2,4 \%$ plus de $20 \%$ de THC. La plus forte teneur observée concernait une saisie effectuée en 2003 dans la région de Bordeaux, avec $21 \%$ de THC.

Les résultats concernant la résine sont indiqués dans la Figure 2. Depuis 1993, le nombre d'échantillons faiblement « dosés » (contenant moins de 5\% de THC) a diminué de manière très significative, passant de $48 \%$ des échantillons en 1993 à $15 \%$ en 2004. Les échan- tillons contenant plus de $20 \%$ de THC restent en 2004 très rarement rencontrés, représentant seulement $2 \%$ des saisies. En revanche, on constate depuis une dizaine d'années une augmentation régulière du nombre d'échantillons de résine contenant entre 10 et $15 \%$ de THC. De $1 \%$ des échantillons en 1993, ils représentaient en $200432 \%$ des saisies. La teneur moyenne observée parmi les 2613 échantillons de résine saisis entre 2001 et 2004 était de 8,84\% alors qu'elle était de 4,36\% pendant la période 1993-1995 (8), ayant donc été multipliée par 2 en 10 ans.

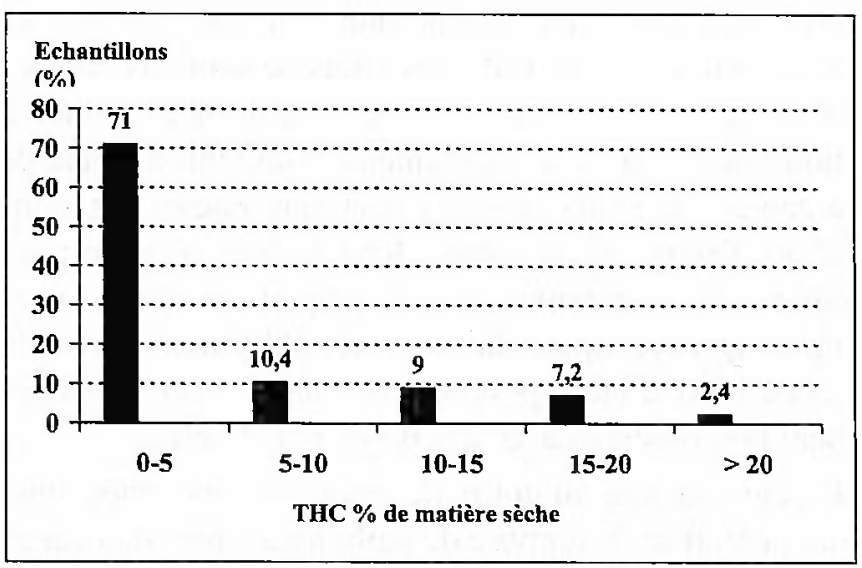

Figure 1 : Teneurs en THC observées en France pour "l'herbe" entre 2001 et 2004.

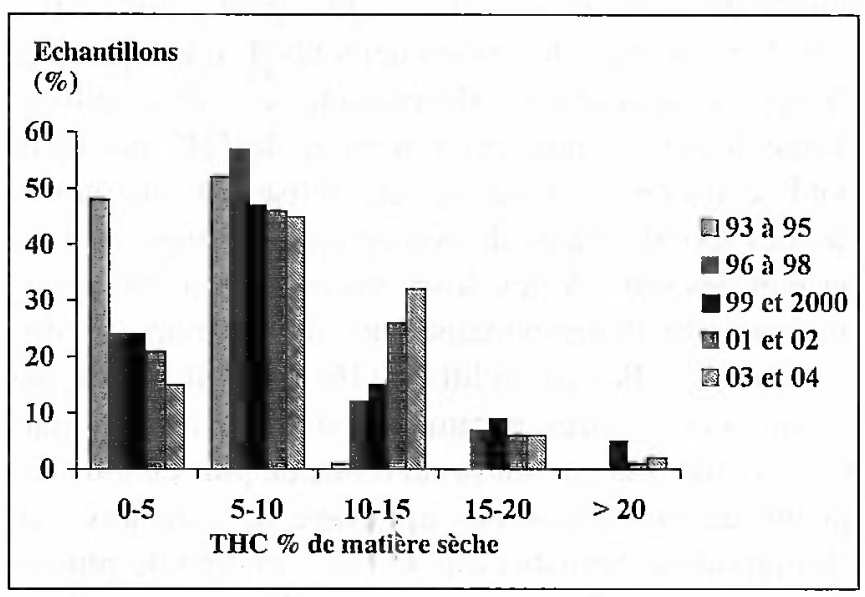

Figure 2 : Évolution des teneurs en THC dans les échantillons de résine entre 1993 et 2004.

\section{Discussion et conclusion}

L'évolution la plus caractéristique concernant l'herbe est l'augmentation du nombre d'échantillons contenant plus de $20 \%$ de THC, qui est passée de $0,5 \%$ en 1993 à $2,4 \%$ en 2004 . Cette observation est à relier avec la présence sur le marché clandestin français de produits provenant des pays du Nord de l'Europe et principalement des Pays-Bas. Grâce à des techniques horticoles très avancées (cultures sous serres, hydroponiques, condi- 
tions de luminosité et de température optimales) et à l'utilisation de graines sélectionnées, les néerlandais réussissent désormais à obtenir des variétés à très forte teneur en THC (5).

Quant à la résine, l'évolution la plus remarquable est le recentrage des teneurs en THC dans la fourchette 10 à $15 \%$. Ce constat se traduit par une teneur moyenne ayant été multipliée par 2 en 10 ans. Ce doublement de la teneur moyenne est certes beaucoup plus modeste que ce qui est souvent annoncé (sans fondement scientifique) dans certains articles de la presse populaire, mais cette évolution est déjà très importante en terme de conséquences. En effet avec des produits « titrant » en moyenne à environ $9 \%$ de THC, les effets ne sont pas comparables à ceux obtenus avec des échantillons titrant à moins de 5\%, avec très certainement un renforcement de la composante hallucinogène. Certains auteurs ont montré que l'usage de cannabis « haut dosage » était responsable d'une augmentation de la plupart des effets somatiques et psychiques du cannabis (11), mais aussi de l'apparition d'états psychotiques aigus (12) généralement non observés avec des doses plus faibles.

Il a été constaté au cours de ces dernières années une augmentation du nombre de pathologies psychiatriques lourdes liées à l'usage de cannabis $(7,13,14)$, d'accidents de la voie publique (15), de suicides ou autres cas médico-légaux. La consommation de plus en plus fréquente de cannabis « haut dosage » peut jouer un rôle dans l'occurrence de ces évènements. L'importance de l'ivresse cannabique est dépendante de la dose utilisée. A une dose moyenne, environ $50 \mu \mathrm{g}$ de THC par kg de poids corporel, l'ivresse se caractérise par une euphorie, des modifications de perception du temps, de l'espace et des sens. A des doses supérieures à $200 \mu \mathrm{g} / \mathrm{kg}$, prédominent dépersonnalisation, déréalisation et distorsions visuelles ou auditives (16). Mais il ne faut pas oublier deux autres paramètres d'importance, jouant aussi un rôle majeur sur la survenue de plus en plus fréquente de problèmes liés à l'usage de cannabis : (i) l'initiation au cannabis qui se fait à un âge de plus en plus précoce et il a été montré que plus un consommateur est jeune, plus grands sont les risques (14) (ii) les changements de modes de consommation avec en particulier l'utilisation de plus en plus fréquente de pipes à eau ou «bangs ». Ces dispositifs permettent d'obtenir une « défonce » plus importante, en augmentant la quantité de THC pénétrant dans les poumons.

En conclusion, l'analyse de 3322 échantillons de résine et d'herbe saisis en France entre 2001 et 2004 et la comparaison des résultats avec ceux obtenus depuis 1993, a montré que les teneurs en THC n'ont cessé d'augmenter. Avec de tels produits, ces mots de Charles Baudelaire trouvent preneurs :
« Verse-nous ton poison pour qu'il nous réconforte ! Nous voulons, tant ce feu nous brûle le cerveau, Plonger au fond du gouffre, Enfer ou Ciel, qu'importe ? Au fond de l'Inconnu pour trouver du nouveau ! »

\section{Références}

1. Barnett G., Licko V., Thompson T. Behavioral pharmacokinetics of marijuana. Psychopharmacology (berl) 1985 ; $85: 51-6$.

2. Harder S., Rietbrock S. Concentration-effect relationship of delta-9-tetrahydrocannabiol and prediction of psychotropic effects after smoking marijuana. Int. J. Clin. Pharmacol. Ther. 1997 ; 35 : 155-9.

3. Lane SS.D., Cherek D.R., Lieving L.M., Tcheremissine O.V. Marijuana effects on human forgetting functions. J. Exp. Anal. Behav. $2005 ; 83: 67-83$.

4. Reynaud M., Parquet P..J., Lagrue G. In : Les pratiques addictives. Paris : Odile Jacob ed. 2000.

5. Paris M., Tran N. The existence of "Nederwiet", a new fact in the history of cannabis. Ann. Pharm. Fr. 1998 ; $56: 264-7$.

6. Rapport de l'Académie des Sciences. Aspects moléculaires, cellulaires et physiologiques des effets du cannabis. $n^{\circ} 39$. Paris : Lavoisier Technique et Documentation, avril 1997.

7. Costentin J. Données neurobiologiques récentes sur le cannabis. Bul. Acad. Natle Med. 2002 ; 186 : 47-57.

8. Mura P., Perrin M., Chabrillat M., Chaudron H., Dumestre-Toulet V., Barc S., Pépin G. L'augmentation des teneurs en delta- 9 tétrahydrocannabinol dans les produits à base de cannabis en France : mythe ou réalité ? Ann. Toxicol. Anal. $2001 ; 13: 75-9$.

9. Hall W., Swift W. The THC content of cannabis in Australia: evidence and implications. Aust. N. Z. J. Public Health $2000 ; 24: 503-8$.

10. Stambouli H., El Bouri A., Bellimam M.H., Bouayoun T., El Karni N. Concentrations du delta-9 THC dans les cultures de Cannabis sativa L. du. nord du Maroc. Ann. Toxicol. Anal. $2005 ; 17$ : 79-86.

11. Chait L.D., Burke K.A. Preference for high- versus lowpotency marijuana. Pharmacol. Biochem. Behav. 1994 ; $49: 643-7$

12. Schlimme J., Rada D., Schneider U. Cannabis consumption and its psychosocial effects in a comparison of different cultures. Fortschr. Neurol. Psychiatr. $2001 ; 69: 367-73$.

13, de Irala J., Ruiz-Canela M., Martinez-Gonzalez M.A. Causal relationship between cannabis use and psychotic symptoms or depression. Should we wait and see ? A public health perspective. Med. Sci. Monit. 2005 ; 24 : 355-8.

14. Expertise collective INSERM. Cannabis. Quels effets sur le comportement et la santé ? In : INSERM, ed. Paris : Jouve, 2001.

15. Mura P., Chatelain C., Dumestre V., Gaulier J.M., Ghysel M.H., Lacroix C., Kergueris M.F., Lhermitte M., Moulsma M., Pépin G., Vincent F., Kintz P. Use of drugs of abuse in less than 30-year-old drivers killed in a road crash in France: a spectacular increase for cannabis, cocaine and amphetamines. Forensic Sci. Int. 2006 ; sous presse.

16. Ghodse A.H. Cannabis psychosis. Br. J. Addict. 1986 ; $81: 473-8$. 